\title{
Prognostic implications of pericardial and pleural effusion in patients with cardiac amyloidosis
}

\author{
Christina Binder ${ }^{1} \cdot$ Franz Duca $^{1} \cdot$ Thomas Binder $^{1} \cdot$ René Rettl $^{1} \cdot$ Theresa Marie Dachs $^{1} \cdot$ Benjamin Seirer ${ }^{1}$. \\ Luciana Camuz Ligios $^{1} \cdot$ Fabian Dusik $^{1}$. Christophe Capelle ${ }^{1} \cdot$ Hong Qin $^{1} \cdot$ Hermine Agis $^{2} \cdot$ Renate Kain $^{3}$. \\ Christian Hengstenberg ${ }^{1} \cdot$ Roza Badr Eslam $^{1} \cdot$ Diana Bonderman $^{1}{ }^{10}$
}

Received: 23 April 2020 / Accepted: 25 June 2020 / Published online: 10 September 2020

(c) The Author(s) 2020

\begin{abstract}
Background Pericardial and pleural effusion are common findings in patients with cardiac amyloidosis (CA). It is not known, whether effusions correlate with right ventricular (RV) function in these patients. Furthermore, data on the prognostic significance of pleural and pericardial effusion in CA is scarce.

Methods Patients with transthyretin (ATTR) and light chain (AL) CA were included in a clinical registry. All patients underwent transthoracic echocardiography at baseline. The presence of pericardial and pleural effusion was determined in every patient. The clinical endpoint was defined as cardiac death or heart failure hospitalization.

Results In total, 143 patients were analysed. Of these, 85 patients were diagnosed with ATTR and 58 patients with AL. Twenty-four patients presented with isolated pericardial effusion and 35 with isolated pleural effusion. In 19 patients, both pericardial and pleural effusion were found and in 65 patients no effusion was present at baseline. The presence of pleural effusion correlated well with poor RV function, measured by global RV free-wall strain $(p=0.007)$ in patients with AL, but not in ATTR. No such correlation could be found for pericardial effusion in either amyloidosis subtype. Patients with AL presenting with pleural effusion had worse outcomes compared to patients with pericardial effusion alone or no effusion at baseline. In the ATTR group, there was no difference in outcomes according to presence and type of effusion.

Conclusion More than 50\% of patients with CA presented with pleural and/or pericardial effusions. While pleural effusion was clearly associated with poor RV function in AL, we were not able to detect this association with pericardial effusion.
\end{abstract}

Keywords Cardiac amyloidosis $\cdot$ Echocardiography $\cdot$ Effusion $\cdot$ Right ventricle $\cdot$ Speckle tracking imaging $\cdot$ Strain $\cdot$ Outcome

Electronic supplementary material The online version of this article (https://doi.org/10.1007/s00392-020-01698-7) contains supplementary material, which is available to authorized users.

\footnotetext{
Roza Badr Eslam

roza.badreslam@meduniwien.ac.at

Diana Bonderman

diana.bonderman@meduniwien.ac.at

1 Department of Internal Medicine II, Department of Cardiology, Medical University of Vienna, Waehringer Guertel 18-20, 1090 Vienna, Austria

2 Department of Internal Medicine I, Department of Oncology, Medical University of Vienna, Vienna, Austria

3 Clinical Institute of Pathology, Medical University of Vienna, Vienna, Austria
}

\section{Introduction}

Pleural and pericardial effusions are often seen in patients with heart failure (HF) and are generally attributed to right heart failure [1]. Effusions are also especially common and occur in more than $50 \%$ of patients with cardiac amyloidosis (CA) [2, 3]. However, data on the exact prevalence and relevance of effusions in CA are scarce and descriptions are largely limited to small single centre studies and case reports. $[4,5]$. 


\section{Pericardial effusions in cardiac amyloidosis}

Ultrasound is an ideal imaging modality to detect pericardial and pleural effusion as it is more sensitive than physical examination or chest X-ray [6]. It is, therefore, suggested in the current guidelines as a standard assessment in the evaluation of patients with HF and should, therefore, also be part of a detailed echocardiographic examination in patients with CA [7]. One study reported on the predictive effect of pericardial effusion in patients with light chain amyloidosis (AL); however, the studied cohort was small $(n=31)$ and only analysed the effects of effusion on all-cause death rather than cardiac death $[8,9]$. There has recently been evidence that pericardial effusion may also be a sign of myocardial edema following local amyloid infiltration and consecutive inflammation in patients with AL and ATTR [10,11]. This may be especially relevant in AL due to cytotoxicity of light chain amyloid fibrils [12]. On this note, Riduoani et al. were recently able to show that cardiac magnetic resonance imaging (CMR) could detect local inflammation using T2 weighted sequences and may be useful in distinguishing between ATTR and AL [13]. It has also previously been proposed that in patients with AL, pleural effusion may not only result from right HF, but also from primary pleural infiltration causing consecutive fluid secretion [14]. Another potential mechanism could be a fluid shift resulting from a decrease in intravascular oncotic pressure due to an increased renal loss or reduced production of serum proteins. The exact mechanism of the development of pericardial and pleural effusions in CA even in patients with presumably preserved left and right ventricular (LV, RV) function, however, remains unclear.

We conducted this study to further shed light on pericardial and pleural effusions in AL and ATTR with cardiac involvement and their impact on cardiac outcome.

\section{Methods}

\section{Study population}

We consecutively included patients with AL and ATTR amyloidosis in our prospective clinical registry at the Medical University of Vienna. Diagnosis as well as follow-up were performed and documented at our dedicated CA outpatient clinic. Visits were routinely scheduled every 6 months or more frequently when appropriate, as judged by the clinician. When necessary, patients were admitted to the cardiology ward for intensified treatment. All patients gave written informed consent before study inclusion. The study protocol complies with the Declaration of Helsinki and was approved by the local Ethics Committee of the Medical University of Vienna, Austria (Ethics committee identification number: 796/2010).

\section{Diagnosis of cardiac amyloidosis}

In the years preceding 2016, the diagnosis of ATTR was made by endomyocardial biopsy (EMB). At least five tissue samples were taken from the LV myocardium (Bipal® biopsy foceps Cordis ${ }^{\circledR}$ Corporation, Bridgewater, NJ). Specimens were then fixed in formaldehyde, embedded in paraffin and subsequently stained with congo-red dye to detect interstitial amyloid deposits. Additionally, tissue samples were examined under polarized light to reveal green birefringence. When amyloid fibrils were found, inmmunohistochemical analysis was performed to further characterize amyloid type according to local standard established procedures (AmY-kit amyloid antibodies, Martinsried, Germany).

Following the landmark publication of Gillmore et al. in 2016, which presented a non-invasive diagnostic algorithm for the diagnosis of ATTR using serum and urine light chain analysis as well as bone scintigraphy, transthoracic echocardiography (TTE) and/or CMR, EMB was only performed when non-invasive test results, where ambiguous or unclear [15]. CMR was performed including gadolinium contrast application, T1 mapping and calculation of extracellular volume, as previously described $[16,17]$.

The diagnosis of AL was either made by EMB or by extramyocardial biopsy. To confirm the presence of cardiac involvement, at least one of the following features had to be present: (1) LV hypertrophy with an interventricular septum (IVS) thickness of $>12 \mathrm{~mm}$ and/or presence of apical sparing determined by TTE or (2) elevated cardiac biomarkers $[18,19]$.

\section{Definitions of clinical endpoints}

Clinical outcomes were documented by follow-up at our outpatient clinic, as well as by phone calls and by screening medical records in our electronic hospital record system. The clinical endpoints were defined as cardiac death and death from any cause. Further endpoints were death from any cause and a combined endpoint of cardiac death or HF hospitalization. In the case of a clinical event, local and external records were carefully screened and cause of death was reviewed by a clinical adjudication committee of board certified cardiology specialists (D.B., R.B.). HF hospitalization was defined as an event leading to sudden dyspnea, weight gain, peripheral edema and requiring admission to hospital and/or intravenous diuretic therapy. 


\section{Baseline assessment and transthoracic echocardiography}

Baseline assessment was performed at our CA outpatient clinic and included demographic, clinical, laboratory and imaging parameters.

All TTE exams included standard imaging parameters, as well as an extended protocol performed by experienced and certified specialists on high-end machines (GE Vivid 95 and Vivid 7; GE Healthcare, Wauwatosa, WI, USA). Images were acquired and measurements were taken according to current guideline recommendations [20-22].

Presence and size of pericardial effusion was assessed in a subcostal four-chamber view. When imaging quality was suboptimal from a subcostal approach, an apical fourchamber view was used to determine the presence of pericardial effusion. Assessment of pleural effusion was performed bilaterally in every patient. Additionally, the presence of effusion was confirmed by chest X-ray or CMR in patients who underwent CMR for the diagnosis of CA.

Speckle tracking imaging was performed after image acquisition on a modern offline clinical workstation equipped with dedicated software (EchoPAC; GE Healthcare, Wauwatosa, WI, USA). The global longitudinal strain of the LV (LV-GLS) was measured by tracking the myocardium in an optimized apical three- four- and two-chamber view. The global longitudinal strain of the RV (RV-GLS) was measured in the free lateral RV wall in an optimized apical four-chamber view. All obtained TTE parameters are shown in Table 1.

Pericardial effusion size was measured at the location of maximum fluid accumulation. Pericardial effusions were defined as hemodynamically significant according to current guidelines [4], i.e., presence of swinging heart, early diastolic collapse of the RV, abnormal ventricular septal motion, exaggerated respiratory variability $(>25 \%)$ in mitral inflow velocity, or when the patient was clinically compromised (cardiogenic shock).

\section{Statistical analysis}

Continuous data are shown as median values and interquartile range (IQR). Categorical variables are expressed as numbers and percentages. Analysis of variance (ANOVA), Student's $T$-test and Chi square test were used as appropriate to determine statistically significant differences in baseline variables. Univariable Cox regression analysis was applied to test baseline variables for effects on the primary endpoint of cardiac death or HF hospitalization. Variables which significantly predicted outcome were then entered in a stepwise forward multivariable Cox regression model within respective categories to adjust for potential confounding effects. Kaplan-Meier plots with respective log-rank tests were computed to assess the time-dependent discriminative power of effusion type on endpoints. A $p$-value $<0.05$ was considered as statistically significant. All analyses were performed using SPSS 24 (IBM Corp. NY, USA).

\section{Results}

\section{Study cohort}

In total, 169 patients with cardiac AL or ATTR amyloidosis were included in our clinical amyloidosis registry between March 2012 and January 2019. However, only 143 patients had sufficient echocardiographic image quality of both ventricles to be included in this study. Of these, 85 patients (59.4\%) presented with ATTR and 58 (40.6\%) with AL. Diagnosis was confirmed by EMB in 19 patients with AL (13.3\%) and 31 patients with ATTR (21.7\%). In 37 patients, the diagnosis of CA was made using a combination of noncardiac biopsy and imaging $(\mathrm{AL}=33$, ATTR =4). All other were diagnosed by imaging alone. CMR was performed in 113 patients $(\mathrm{AL}=47, \mathrm{ATTR}=66)$.

\section{Baseline characteristics and clinical presentation}

Detailed baseline characteristics of the study population according to presence of pericardial and/or pleural effusion are shown in Table 1.

Patients with effusions $(n=78)$ presented with more severe clinical symptoms as described by New York Heart Association (NYHA) class $(p=0.018)$ and were more commonly already on loop diuretics $(p<0.001)$ and/or aldosterone antagonists $(p=0.002)$ at baseline. Left ventricular global longitudinal strain (LV-GLS) as well as RV free wall strain (RV-GLS) were more severely impaired in patients with pericardial or pleural effusions with $p$-values of 0.007 and 0.004 , respectively.

Notably, patients with effusions not only showed higher levels of cardiac biomarkers (median NT-pro BNP $4268.5 \mathrm{pg} / \mathrm{ml}$ (IQR 2048.0-8866.0 pg/mL) versus $1635.0 \mathrm{pg}$ / $\mathrm{ml}$ (IQR 827.0-3549.0 pg/mL), $p<0.001$ and median troponin T $68.0 \mathrm{ng} / \mathrm{L}$ (IQR43.5-118.0 ng/L) versus $28.0 \mathrm{ng} / \mathrm{L}$ (IQR 22.0-56.0 ng/L), $p<0.001$ ), but also higher levels of gamma glutamyltransferase (GGT) of $79.0 \mathrm{U} / \mathrm{L}$ (IQR 46.0-140.0 U/L) versus 42.0 U/L [(IQR26.0-126.0 U/L), $p=0.033]$ and lower levels of serum albumin of $38.7 \mathrm{~g} / \mathrm{L}$ (IQR 33.0-42.4 g/L) versus $43.0 \mathrm{~g} / \mathrm{L}$ [(IQR 38.3-45.2 g/L), $p=0.002]$. Furthermore, median serum lactate dehydrogenase was higher in patients presenting with pericardial and/ or pleural effusions [(241.0 U/L (IQR 206.0-292.5 U/L) versus 224.0 U/L (IQR 190.0-249.0 U/L), $p=0.017$ ]. 
Table 1 Baseline characteristics for patients with amyloidosis with and without pericardial and/or pleural effusion

\begin{tabular}{|c|c|c|c|c|}
\hline & $\begin{array}{l}\text { Total population } \\
(n=143)\end{array}$ & $\begin{array}{l}\text { No effusion } \\
(n=65)\end{array}$ & $\begin{array}{l}\text { Pericardial and/or pleural effusion } \\
(n=78)\end{array}$ & $p$-value \\
\hline \multicolumn{5}{|l|}{ Clinical parameters } \\
\hline Age, years & $73.0(66.0-78.0)$ & $78.0(67.0-78.0)$ & $74.0(63.0-80.0)$ & 0.697 \\
\hline Male sex & $107(75)$ & $51(79)$ & $56(72)$ & 0.360 \\
\hline BMI, $\mathrm{kg} / \mathrm{m}^{2}$ & $25.6(23.2-28.4)$ & $26.0(23.0-29.0)$ & $26.0(23.0-28.0)$ & 0.680 \\
\hline Systolic BP, mmHg & $126.5(113.0-140.0)$ & $125.0(113.0-140.0)$ & $128.0(113.0-139.0)$ & 0.654 \\
\hline Diastolic BP, mmHg & $75.0(70.0-85.0)$ & $77.0 / 70.0-87.0)$ & $74.0(69.0-84.0)$ & 0.354 \\
\hline Heart rate, bpm & $75.0(66.5-87.0)$ & $76.0(68.0-87.0)$ & $74.0(65.0-87.0)$ & 0.566 \\
\hline NYHA class & & & & 0.018 \\
\hline NYHA I & $14(9.8)$ & $10(15.4)$ & $4(5.1)$ & \\
\hline NYHA II & $61(42.7)$ & $33(50.8)$ & $28(35.9)$ & \\
\hline NYHA III & $64(44.8)$ & $21(32.3)$ & $43(55.2)$ & \\
\hline NYHA IV & $4(2.7)$ & $1(1.5)$ & $3(3.8)$ & \\
\hline \multicolumn{5}{|l|}{ Comorbidities } \\
\hline Polyneuropathy & $56(39.2)$ & $30(46.2)$ & $26(33.3)$ & 0.118 \\
\hline Diabetes mellitus & $20(14.0)$ & $12(18.5)$ & $8(10.3)$ & 0.168 \\
\hline Atrial fibrillation & $65(45.5)$ & $27(41.5)$ & $38(48.7)$ & 0.352 \\
\hline Coronary artery disease & $29(20.3)$ & $11(16.9)$ & $18(23.1)$ & 0.362 \\
\hline Intracardiac device & $19(23.3)$ & $8(12.3)$ & $11(14.1)$ & 0.753 \\
\hline \multicolumn{5}{|l|}{ Concomitant medication } \\
\hline AT II antagonist & $35(24.5)$ & $19(29.2)$ & $16(20.5)$ & 0.227 \\
\hline ACE inhibitor & $37(25.9)$ & $13(20.0)$ & $24(30.8)$ & 0.143 \\
\hline Beta-blocker & $76(53.1)$ & $32(49.2)$ & $44(56.4)$ & 0.392 \\
\hline Aldosterone antagonist & $64(44.8)$ & $20(30.8)$ & $44(56.4)$ & 0.002 \\
\hline Loop diuretics & $91(63.6)$ & $30(46.2)$ & $61(78.2)$ & $<0.001$ \\
\hline Oral anticoagulant & $67(46.9)$ & $29(44.6)$ & $38(48.7)$ & 0.624 \\
\hline \multicolumn{5}{|c|}{ Echocardiography parameters } \\
\hline LA length, mm & $61.0(55.0-68.0)$ & $60.0(55.0-66.0)$ & $63.0(55.0-68.0)$ & 0.147 \\
\hline RA length, mm & $59.0(53.0-64.3)$ & $58.0(53.0-63.0)$ & $60.0(52.0-66.0)$ & 0.236 \\
\hline LVEDD, mm & $41.0(37.0-46.0)$ & $42.0(38.0-47.0)$ & $41.0(34.0-46.0)$ & 0.060 \\
\hline LVEF, \% & $45.0(46.0-64.3)$ & $52.0(47.0-65.0)$ & $55.0(46.0-64.0)$ & 0.993 \\
\hline LV-GLS, - $\%$ & $12.0(15.0-9.0)$ & $13.7(16.8-10.0)$ & $11.3(13.8-8.6)$ & 0.007 \\
\hline IVS, mm & $19.0(16.0-22.0)$ & $18.0(16.0-22.0)$ & $20.0(17.0-23.0)$ & 0.155 \\
\hline RVEDD, mm & $33.0(28.0-38.0)$ & $34.0(29.0-38.0)$ & $33.0(28.0-38.0)$ & 0.496 \\
\hline RV-TDI, m/s & $0.11(0.09-0.14)$ & $0.12(0.09-0.14)$ & $0.11(0.09-0.13)$ & 0.485 \\
\hline TAPSE, mm & $16.0(13.0-19.0)$ & $17.0(14.0-20.0)$ & $15.0(12.0-18.0)$ & 0.055 \\
\hline RV-GLS, - \% & $16.3(20.7-11.3)$ & $17.7(23.0-13.3)$ & $14.0(18.5-10.0)$ & 0.004 \\
\hline TR velocity, $\mathrm{m} / \mathrm{s}$ & $2.9(2.6-3.3)$ & $2.9(2.5-3.2)$ & $2.9(2.7-3.3)$ & 0.446 \\
\hline sPAP, mmHg & $43.0(37.0-58.0)$ & $43.0(32.0-55.0)$ & $43.0(38.0-58.0)$ & 0.528 \\
\hline IVC diameter, mm & $20.0(17.0-23.0)$ & $19.0(14.0-22.0)$ & $20.0(18.0-24.0)$ & 0.237 \\
\hline \multicolumn{5}{|l|}{ Laboratory parameters } \\
\hline NT-pro BNP, pg/mL & $2815.0(1412.0-7173.0)$ & $1635.0(827.0-3549.0)$ & $4268.5(2048.0-8866.0)$ & $<0.001$ \\
\hline Troponin T, ng/L & $52.0(31.0-96.5)$ & $38.0(22.0-56.0)$ & $68.0(43.5-118.0)$ & $<0.001$ \\
\hline Hemoglobin, g/dL & $12.7(11.3-14.2)$ & $12.9(11.3-14.4)$ & $12.7(11.1-14.1)$ & 0.377 \\
\hline Creatinine, $\mathrm{mg} / \mathrm{dL}$ & $1.2(1.0-1.6)$ & $1.3(0.9-1.6)$ & $1.2(1.0-1.6)$ & 0.813 \\
\hline $\mathrm{eGFR}, \mathrm{mL} / \mathrm{min}$ & $51.7(38.3-63.1)$ & $52.3(40.7-72.4)$ & $51.0(37.9-59.1)$ & 0.327 \\
\hline ASAT, U/L & $28.0(22.0-34.0)$ & $27.0(22.0-33.0)$ & $29.0(23.0-35.0)$ & 0.102 \\
\hline ALAT, U/L & $24.0(17.0-33.0)$ & $23.0(17.0-29.0)$ & $24.0(17.0-35.0)$ & 0.424 \\
\hline Albumin, g/L & $40.5(35.0-44.1)$ & $43.3(38.3-45.2)$ & $38.7(33.0-42.4)$ & 0.002 \\
\hline GGT, U/L & $65.0(32.0-137.0)$ & $42.0(26.0-126.0)$ & $79.0(46.0-140.0)$ & $\mathbf{0 . 0 3 3}$ \\
\hline
\end{tabular}


Table 1 (continued)

\begin{tabular}{|c|c|c|c|c|}
\hline & $\begin{array}{l}\text { Total population } \\
(n=143)\end{array}$ & $\begin{array}{l}\text { No effusion } \\
(n=65)\end{array}$ & $\begin{array}{l}\text { Pericardial and/or pleural effusion } \\
(n=78)\end{array}$ & $p$-value \\
\hline $\mathrm{LDH}, \mathrm{U} / \mathrm{L}$ & $231.0(197.0-282.0)$ & $224.0(190.0-249.0)$ & $241.0(206.0-292.5)$ & 0.017 \\
\hline $\mathrm{CRP}, \mathrm{mg} / \mathrm{dL}$ & $0.3(0.1-1.1)$ & $0.2(0.1-0.9)$ & $0.4(0.1-1.1)$ & 0.060 \\
\hline \multicolumn{5}{|c|}{ Cardiac magnetic resonance imaging parameters } \\
\hline IVS, mm & $19.0(15.5-22.0)$ & $18.0(15.0-22.0)$ & $17.0(19.0-22.0)$ & 0.197 \\
\hline LVEF, \% & $57.0(49.0-63.0)$ & $57.0(45.0-65.0)$ & $57.0(50.0-62.0)$ & 0.714 \\
\hline LV-ECV, \% & $46.0(40.0-55.5)$ & $45.0(36.0-52.0)$ & $49.0(41.0-58.0)$ & 0.090 \\
\hline RVEF, \% & $49.5(40.1-60.5)$ & $49.0(42.0-61.0)$ & $51.0(38.0-59.0)$ & 0.556 \\
\hline
\end{tabular}

Continuous variables are given in median and interquartile range, categorical data are shown as numbers and percentages

$B M I$ body mass index, $B P$ blood pressure, $b p m$ beats per minute, $N Y H A$ New York Heart Association, $C A D$ coronary artery disease, $P M$ pace maker, ICD intracardiac defibrillator, $A T$ II angiotensin II, ACE angiotensin converting enzyme, $L A$ left atrium, $R A$ right atrium, $L V E D D$ left ventricular end-diastolic diameter, $L V E F$ left ventricular ejection fraction, $I V S$ intraventricular septum, $R V E D D$ right ventricular end-diastolic diameter, $R V$-TDI right ventricular tissue Doppler index, TAPSE tricuspid annular plane systolic excursion, $R V$ - $G L S$ right ventricular global longitudinal strain, $T R$ tricuspid regurgitation $S P A P$ systolic pulmonary artery pressure, $I V C$ inferior vena cava, $N T$-pro BNP N-terminal pro brain natriuretic peptide, $e G F R$ estimated glomerular filtration rate, $A S A T$ aspartate aminotransferase, $A L A T$ alanine aminotransferase, $G G T$ gamma glutamyltransferase, $L D H$ lactate dehydrogenase, $C R P C$-reactive protein, $L V$ - $E C V$ left ventricular extra cellular volume, $R V E F$ right ventricular ejection fraction

Table 2 Effusions in patients with cardiac light-chain (AL) versus transthyretin amyloidosis (ATTR)

\begin{tabular}{llll}
\hline & $\begin{array}{l}\text { Total population } \\
(n=143)\end{array}$ & $\begin{array}{l}\text { AL } \\
(n=58)\end{array}$ & $\begin{array}{l}\text { ATTR } \\
(n=85)\end{array}$ \\
\hline No effusion & $65(45.5)$ & $21(36.2)$ & $44(51.8)$ \\
$\begin{array}{l}\text { Isolated pleural effusion } \\
\text { Isolated pericardial effusion, }\end{array}$ & $35(24.5)$ & $16(27.6)$ & $19(22.4)$ \\
$\begin{array}{l}\text { Pleural and pericardial } \\
\text { effusion }\end{array}$ & $19(16.8)$ & $12(20.7)$ & $12(14.1)$ \\
\hline
\end{tabular}

Values are given as numbers and percentages.

\section{Pericardial and pleural effusion}

At baseline, 35 patients $(24.5 \%)$ presented with isolated pleural effusion, 24 patients $(16.8 \%)$ with isolated pericardial effusion and 19 patients (13.3\%) with both pleural and pericardial effusion. Pleural effusions detected by echocardiography could only be confirmed by chest X-ray in $76.2 \%$ of all patients. Table 2 shows a detailed description of types of effusions and their distribution in patients with $\mathrm{AL}$ versus ATTR. In general, pericardial effusions were small with a medium diameter of $7.1 \mathrm{~mm}$ (IQR 5.2-9.7 $\mathrm{mm}$ ) and none were hemodynamically compromising.

\section{Effusions in light chain amyloidosis}

When analysing parameters associated with right heart dimensions and function, we found that patients with $\mathrm{AL}$ presenting with pleural effusions at baseline had more impaired RV function, which was reflected by RV-GLS, TAPSE and RV-TDI. There was no difference in RV strain when comparing the basal- and mid segments with the apical segments of the RV (Fig. 1a). Patients with pleural effusions had higher amyloid burden, seen as increased interventricular wall thickness (IVS) and LV extra cellular volume (LV-ECV) resulting in impaired contractility as measured by LV-GLS. Interestingly, not only cardiac biomarkers such as serum NT-pro BNP and troponin T, but also GGT and CRP levels were higher in patients with pleural effusions (Table 3).

By contrast, AL patients presenting with pericardial effusions only showed significantly more impaired strain in the basal and mid regions of the RV. Apical RV regions also showed more impaired function, even though this difference was not statistically significant (supplementary table S1). Notably, there was no difference in cardiac biomarkers between the two groups.

\section{Effusions in transthyretin amyloidosis}

In patients diagnosed with ATTR, the presence of pleural effusions was not accompanied by more impaired RV strain values or other parameters of RV function. However, when comparing the basal and mid-segments of the RV to the apical segments, we were able to detect that the more apical segments showed better RV function (RV-apical sparing, Fig. 1b). Similarly, there was no statistically significant association between the presence of pleural effusion and LV-function or degree of LV amyloid infiltration seen in CMR. Nevertheless, serum levels of cardiac biomarkers were significantly higher in patients with pleural effusions compared to those without. Notably, albumin levels were lower in patients presenting with pleural effusions (Table 4). Supplementary table S2 shows, that there is no association 

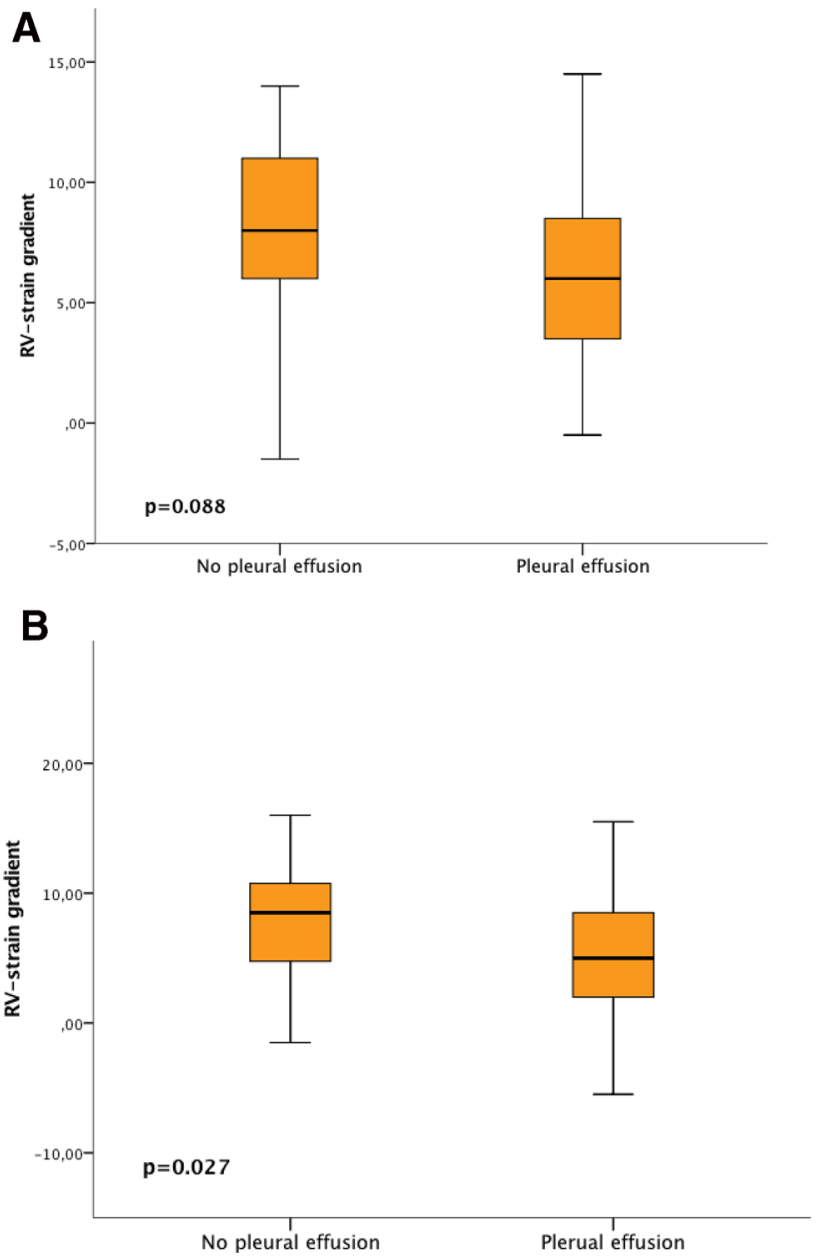

Fig. 1 2D strain in the basal and mid segments compared to the apical segments of the right ventricle in light-chain amyloidosis (a) and ATTR (b) in patients with and without pleural effusions

between RV function and pericardial effusion in patients with ATTR.

\section{Left and right heart function in patients with $A L$ versus ATTR}

LV hypertrophy was more pronounced in patients with ATTR than those who had been diagnosed with AL with a mean IVS thickness of $20 \mathrm{~mm}$ versus $17 \mathrm{~mm}$, respectively. $\mathrm{LV}$ function was better in patients with $\mathrm{AL}$ as indicated by LV-GLS ( $p=0.010)$, as well as LV ejection fraction (LVEF) measured by CMR $(p<0.001)$. Interestingly, RV function measured using the volumetric method of RV ejection fraction by CMR was also better in patients with $\operatorname{AL}(p=0.007)$, while RV function parameters reflecting RV contractility, such as RV-GLS and TAPSE did not show any differences between patients with AL and ATTR ( $p=0.562$ and 0.298, respectively).

\section{Outcomes according to presence and localization of effusion}

After a median follow-up time of 19.0 months (IQR 9.5-32.5), 42 patients had died (29.4\%). In more detail, 28 patients with $\mathrm{AL}$ (48.3\% of the total AL population) and 14 patients with ATTR (16.5\% of the total ATTR population) died. During follow-up, 26 (61.9\%) could be classified as cardiac deaths. In seven patients, the exact cause of death could not be certified, due to lack of medical records or information from relatives and were, therefore, classified as all-cause death.

Kaplan-Meier analysis showed that among the total population of cardiac amyloidosis, patients with pleural effusions had worse outcomes than patients with no effusions or isolated pericardial effusions. This was irrespective of concomitant presence of pericardial effusion in addition to pleural effusion. Furthermore, the presence of isolated pericardial effusion did not have an impact on outcome. When separating the studied cohort by amyloid subtype, we saw that effusion type was predictive for outcome in patients with AL, but not in ATTR (Fig. 2). In addition, we used univariable Cox regression models to calculate hazard ratios (HR) for cardiac death for all baseline parameters in patients with AL. We found that the presence of pleural effusion predicted adverse outcome with a HR of 5.604 [(2.334-13.456), $p<0.001]$ alongside with NYHA class, NT-pro BNP and troponin T. Among echocardiography parameters, LV global longitudinal strain (LV-GLS), LV end diastolic diameter (indexed to body surface area), interventricular septum thickness, and RV tissue Doppler index (RV-TDI) predicted outcome in the univariable model. After adjusting for confounding factors within respective categories of baseline characteristics, only NYHA class, LV-GLS and NT-pro BNP remained predictive in the multivariable model (Table 5).

\section{Discussion}

Our data show that the presence of pleural effusion in patients with AL is associated with poor RV function and higher serum levels of NT-pro BNP and troponin T. While cardiac biomarkers were also higher in patients with ATTR and pleural effusion, there was no difference in RV function depending on the presence or absence of pleural effusion in these patients. We found that patients with AL presenting with pleural effusions had poor clinical outcomes and that concomitant or isolated pericardial effusions were not associated with worse outcomes in these patients.

Few previous studies have described the effects of effusions on outcome and even less considered both pericardial and pleural effusions in CA. Berk et al. previously proposed that pleural effusion may not be an effect of right 
Table 3 Baseline echocardiographic and laboratory parameters of patients with light chain amyloidosis $(n=58)$ with and without pleural effusion* (extracted)

\begin{tabular}{|c|c|c|c|}
\hline & No pleural effusion $(n=33)$ & $\begin{array}{l}\text { Pleural effusion } \\
(n=25)\end{array}$ & $p$-value \\
\hline \multicolumn{4}{|c|}{ Echocardiography parameters } \\
\hline LA length mm & $60.0(56.0-67.0)$ & $60.0(52.0-64.0)$ & 0.665 \\
\hline LVEDD, mm & $46.0(43.0-48.0)$ & $35.0(32.0-46.0)$ & $<0.001$ \\
\hline LVEF, \% & $61.0(51.0-70.0)$ & $56.0(46.0-65.0)$ & 0.626 \\
\hline LV-GLS, - \% & $14.8(19.10-14.0)$ & $11.3(14.8-8.3)$ & $\mathbf{0 . 0 0 3}$ \\
\hline IVS, mm & $16.0(14.0-18.0)$ & $19.0(16.0-22.0)$ & 0.170 \\
\hline $\mathrm{RV}$ diameter, mm & $34.0(30.0-37.0)$ & $30.0(26.0-36.0)$ & 0.044 \\
\hline RA length, mm & $59.0(51.0-64.0)$ & $58.0(52.0-61.0)$ & 0.826 \\
\hline RV strain basal, $-\%$ & $16.5(20.0-14.0)$ & $13.0(18.0-11.0)$ & 0.009 \\
\hline RV strain mid, -\% & $18.0(21.0-15.0)$ & $14.0(17.0-11.0)$ & 0.007 \\
\hline RV strain apical, - \% & $19.0(23.0-15.0)$ & $14.0(18.0-12.0)$ & 0.013 \\
\hline RV- GLS, - \% & $18.0(21.3-15.0)$ & $14.0(17.7-11.0)$ & 0.007 \\
\hline RV- TDI, m/s & $0.14(0.13-0.16)$ & $0.11(0.09-0.12)$ & 0.009 \\
\hline TAPSE, mm & $18.0(15.0-22.0)$ & $14.0(13.0-16.0)$ & 0.001 \\
\hline TR velocity, $\mathrm{m} / \mathrm{s}$ & $3.0(2.6-3.7)$ & $2.8(2.6-3.1)$ & 0.251 \\
\hline sPAP, mmHg & $51.0(35.0-67.0)$ & $42.5(39.0-50.0)$ & 0.354 \\
\hline IVC diameter, mm & $19.0(14.0-25.0)$ & $19.0(18.0-23.0)$ & 0.594 \\
\hline \multicolumn{4}{|l|}{ Laboratory parameters } \\
\hline NT-pro BNP, pg/mL & $2292.0(1121.0-4118.0)$ & $8866.0(4751.0-17,103.0)$ & $<0.001$ \\
\hline Troponin T, ng/L & $41.0(21.0-73.5)$ & $112.0(65.0-232.0)$ & 0.001 \\
\hline Gamma GT, U/L & $36.0(21.0-77.0)$ & $117.0(56.0-194.0)$ & $<0.001$ \\
\hline ASAT, U/L & $23.0(18.0-28.0)$ & $27.0(20.0-43.0)$ & 0.094 \\
\hline ALAT, U/L & $21.0(17.0-26.0)$ & $22.0(15.0-43.0)$ & 0.994 \\
\hline Albumin, U/L & $39.2(30.6-42.6)$ & $33.9(28.5-40.3)$ & 0.253 \\
\hline $\mathrm{CRP}, \mathrm{mg} / \mathrm{dl}$ & $0.2(0.1-1.0)$ & $0.9(0.3-1.9)$ & 0.021 \\
\hline $\mathrm{eGFR}, \mathrm{mL} / \mathrm{min} / 1.73 \mathrm{~m}^{2}$ & $47.8(33.3-61.5)$ & $40.5(22.7-55.9)$ & 0.316 \\
\hline \multicolumn{4}{|c|}{ Cardiac magnetic resonance imaging parameters } \\
\hline IVS, mm & $14.0(12.0-26.0)$ & $19.0(15.0-21.0)$ & 0.005 \\
\hline LVEF, \% & $63.0(53.0-66.0)$ & $61.0(57.0-65.0)$ & 0.572 \\
\hline LV-ECV, \% & $40.0(32.0-43.0)$ & $48.0(41.0-59.0)$ & 0.003 \\
\hline RVEF, \% & $57.0(48.0-62.0)$ & $56.0(45.0-5620)$ & 0.405 \\
\hline
\end{tabular}

Continuous variables are given in mean and interquartile range

$L A$ indicates left atrium, $L V E D D$ left ventricular end-diastolic diameter, $L V E F$ left ventricular ejection fraction, $I V S$ interventricular septum, $R V$ right ventricle, $R A$ right atrium, $R V-G L S$ right ventricular global longitudinal strain, TDI tissue Doppler index, TAPSE tricuspid annular plane systolic excursion, TR tricuspid regurgitation, $S P A P$ systolic pulmonary artery pressure, $I V C$ inferior vena cava, NT-pro BNP N-terminal pro brain natriuretic peptide, Gamma GT gamma glutamyltransferase, ASAT aspartate aminotransferase, $A L A T$ alanine aminotransferase, $C R P$ C-reactive protein, $e G F R$ estimated glomerular filtration rate calculated by the modification of diet in renal disease (MDRD) formula, $L V-E C V$ left ventricular extra cellular volume, RVEF right ventricular ejection fraction heart failure, as their data showed no correlation of right heart parameters and presence of pleural effusions in their pure AL cohort [14]. In contrast, we did see a relationship between echocardiographically measured right heart parameters and pleural effusions in patients with AL. The reason for these discordances may be better image quality almost 16 years later and development of more sensitive imaging parameters such as 2D-strain imaging, which were not measured in the mentioned study and could potentially detect more subtle forms of RV dysfunction [23].
Interestingly, our data showed that the association between pleural effusion and RV function was only present in AL, but not in ATTR. However, patients with ATTR and pleural effusions had significantly lower serum albumin levels compared to patients without pleural effusions (Table 3 ). This finding suggests that pleural effusions may develop as a result of decreased plasma oncotic pressure in patients with ATTR, rather than from RV failure and could point to a difference in pathophysiologic mechanisms of effusion in different subtypes of CA. 
Table 4 Baseline echocardiographic and laboratory parameters of patients with transthyretin amyloidosis $(n=85)$ with and without pleural effusion*

\begin{tabular}{|c|c|c|c|}
\hline & No pleural effusion $(n=56)$ & Pleural effusion $(n=29)$ & $p$-value \\
\hline \multicolumn{4}{|c|}{ Echocardiography parameters } \\
\hline LA length, mm & $60.0(54.0-63.0)$ & $66.0(60.0-71.0)$ & 0.036 \\
\hline LVEDD, mm & $40.0(38.0-44.0)$ & $42.0(38.0-45.0)$ & 0.110 \\
\hline LVEF, \% & $52.0(46.0-62.0)$ & $54.0(45.0-63.0)$ & 0.516 \\
\hline LV-GLS, - \% & $12.1(15.3-8.9)$ & $11.2(13.1-8.6)$ & 0.052 \\
\hline IVS, mm & $20.0(17.0-23.0)$ & $20.0(17.0-24.0)$ & 0.790 \\
\hline $\mathrm{RV}$ diameter, mm & $32.0(28.0-37.0)$ & $36.0(31.0-42.0)$ & 0.401 \\
\hline RA length, mm & $58.0(53.0-63.0)$ & $63.0(59.0-68.0)$ & 0.017 \\
\hline RV strain basal, $-\%$ & $17.0(21.0-11.5)$ & $12.5(19.5-10.0)$ & 0.242 \\
\hline RV strain mid, $-\%$ & $17.0(21.0-12.0)$ & $14.0(19.0-10.0)$ & 0.308 \\
\hline RV strain apical, - $\%$ & $17.0(21.0-12.0)$ & $16.5(20.5-10.5)$ & 0.795 \\
\hline RV- GLS, - \% & $17.0(20.8-12.0)$ & $13.8(19.5-10.2)$ & 0.399 \\
\hline RV- TDI, m/s & $0.10(0.08-0.13)$ & $0.10(0.09-0.12)$ & 0.965 \\
\hline TAPSE, mm & $16.0(12.0-19.0)$ & $16.0(11.0-20.0)$ & 0.892 \\
\hline TR velocity, $\mathrm{m} / \mathrm{s}$ & $2.9(2.6-3.2)$ & $2.9(2.6-3.3)$ & 0.783 \\
\hline sPAP, mmHg & $42.0(35.0-55.0)$ & $50.0(37.0-61.0)$ & 0.343 \\
\hline IVC diameter, mm & $18.0(15.0-22.0)$ & $22.0(20.0-25.0)$ & 0.010 \\
\hline \multicolumn{4}{|l|}{ Laboratory parameters } \\
\hline NT-pro BNP, pg/mL & $1574.5(846.4-3367.0)$ & $3908.0(2048.0-7173.0)$ & $<0.001$ \\
\hline Troponin T, ng/L & $39.0(29.0-64.0)$ & $68.0(40.0-79.0)$ & 0.049 \\
\hline Gamma GT, U/L & $66.0(28.5-140.0)$ & $72.0(45.0-140.0)$ & 0.384 \\
\hline ASAT, U/L & $30.0(24.0-34.0)$ & $31.0(25.0-35.0)$ & 0.366 \\
\hline ALAT, U/L & $25.5(20.0-33.0)$ & $23.0(17.0-35.0)$ & 0.450 \\
\hline Albumin, U/L & $43.6(40.4-46.3)$ & $38.7(35.9-42.9)$ & 0.003 \\
\hline $\mathrm{CRP}, \mathrm{mg} / \mathrm{dl}$ & $0.2(0.1-0.7)$ & $0.3(0.2-1.2)$ & 0.096 \\
\hline $\mathrm{eGFR}, \mathrm{mL} / \mathrm{min} / 1.73 \mathrm{~m}^{2}$ & $56.0(45.7-74.7)$ & $47.5(40.6-56.9)$ & 0.092 \\
\hline \multicolumn{4}{|c|}{ Cardiac magnetic resonance imaging parameters } \\
\hline IVS, mm & $18.0(15.0-22.0)$ & $20.0(18.0-23.0)$ & 0.667 \\
\hline LVEF, \% & $55.0(42.0-60.0)$ & $51.0(46.0-57.0)$ & 0.098 \\
\hline LV-ECV, \% & $49.0(43.0-56.0)$ & $51.0(42.0-57.0)$ & 0.173 \\
\hline RVEF, \% & $47.0(39.0-60.0)$ & $48.0(37.0-55.0)$ & 0.474 \\
\hline
\end{tabular}

Continuous variables are given in mean and interquartile range

$L A$ indicates left atrium, $L V E D D$ left ventricular end-diastolic diameter, $L V E F$ left ventricular ejection fraction, $I V S$ interventricular septum, $R V$ right ventricle, $R A$ right atrium, $R V-G L S$ right ventricular global longitudinal strain, TDI tissue Doppler index, TAPSE tricuspid annular plane systolic excursion, $T R$ tricuspid regurgitation, $S P A P$ systolic pulmonary artery pressure, IVC inferior vena cava, NT-pro BNP N-terminal pro brain natriuretic peptide, Gamma GT gamma glutamyltransferase, ASAT aspartate aminotransferase, $A L A T$ alanine aminotransferase, CRP C-reactive protein, $e G F R$ estimated glomerular filtration rate calculated by the modification of diet in renal disease (MDRD) formula, $L V-E C V$ left ventricular extra cellular volume, $R V E F$ right ventricular ejection fraction

*10 patients of the shown population had additional pericardial effusion.
Based on our data, as well as previous studies it can be hypothesized that pleural and pericardial effusions may not simply be explained by the failure of the RV. Effusions could also be a result of local amyloid infiltration of the serosae as well as tissue inflammation. In addition, hypalbuminemia could play a role in the development of pleural effusions in addition to elevated filling pressures [24].

Deeper knowledge of the pathophysiology and prognostic relevance of effusions in $\mathrm{CA}$ is important to guide physicians in their decision whether or not to drain effusions in these patients. While pericardial effusions in our patient cohort were generally small and hemodynamically not relevant, the question of pericardiocentesis is probably not as central as the decision for pleurocentesis in clinical practice. An interesting question which our data do not answer is, if drainage of pleural fluid results in symptom control or change in cardiac or overall prognosis. Since pericardial effusions could only be detected by ultrasound in some cases, we emphasize 
Fig. 2 Kaplan-Meier plot showing time to cardiac death or heart failure in all patients with cardiac amyloidosis $(n=143$,

a), patients with light chain amyloidosis $(n=58, \mathbf{b})$ and transthyretin amyloidosis $(n=85$, c) according to the presence of pericardial and/or pleural effusion at baseline

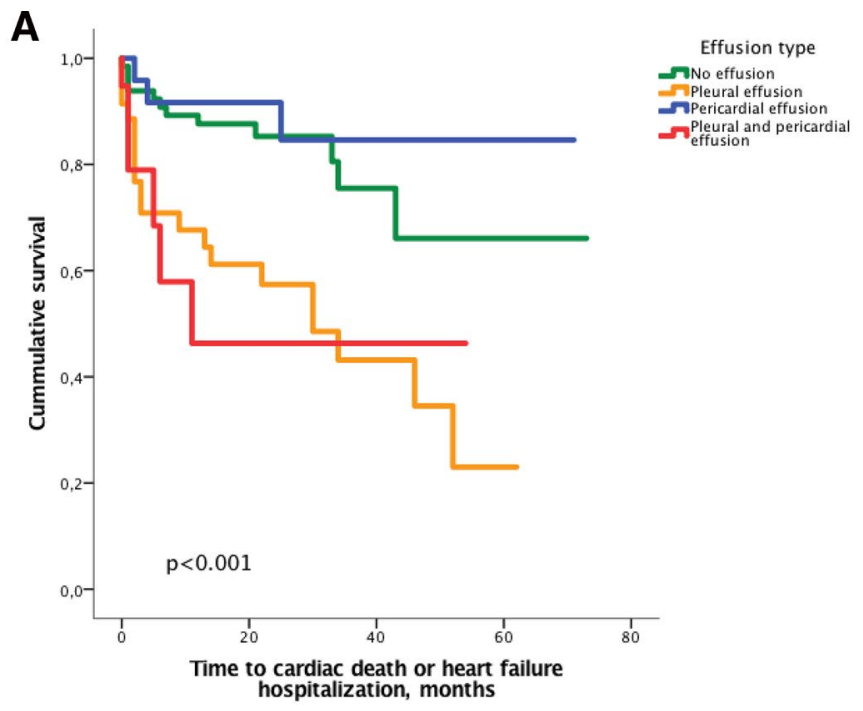

B

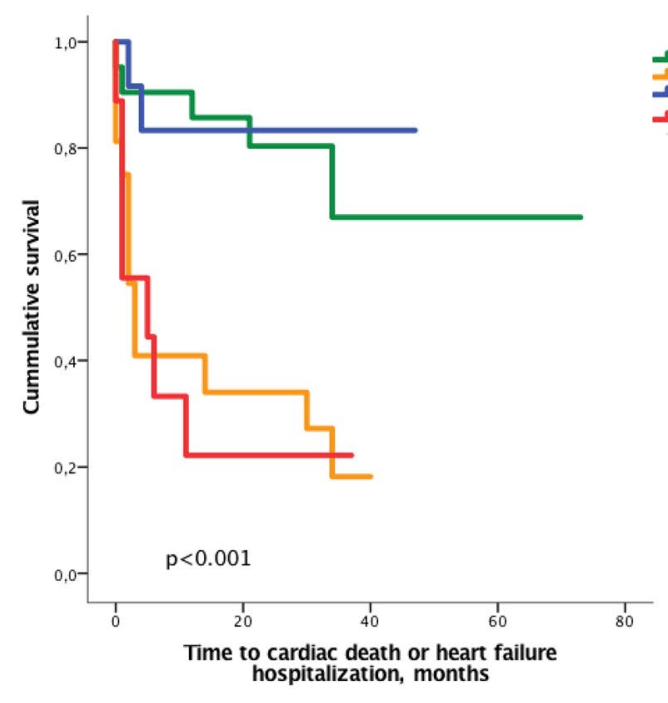

C

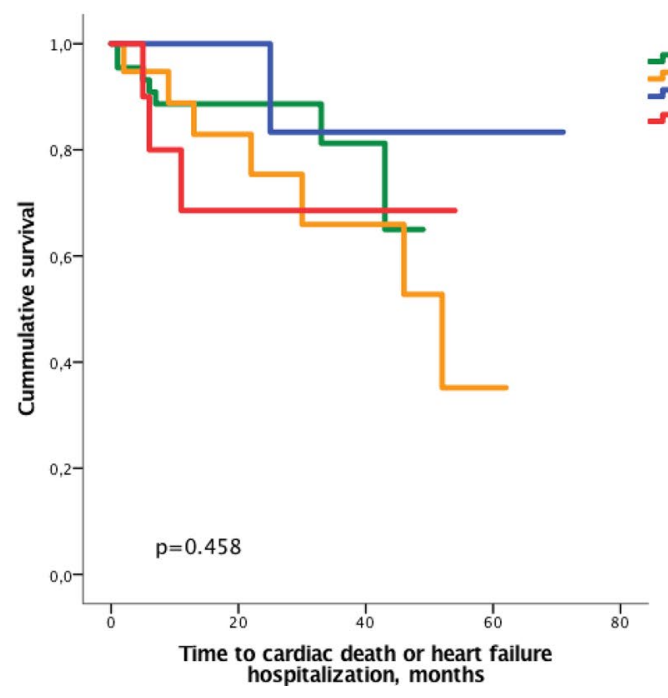

Effusion type INo effusion TPleural effusion Pericardial effusion Pleural and pericardia 
Table 5 Univariable and multivariable Cox regression analysis of baseline characteristics in patients with light chain amyloidosis calculated for the endpoint of cardiac death or heart failure hospitalization

\begin{tabular}{|c|c|c|c|c|}
\hline & Univariable HR (95\%CI) & $p$-value & Multivariable HR (95\%CI) & $p$-value \\
\hline \multicolumn{5}{|l|}{ Clinical variables } \\
\hline Age, years & $0.999(0.997-1.001)$ & 0.270 & & \\
\hline Male sex & $0.465(0.214-1.013)$ & 0.054 & & \\
\hline BMI, $\mathrm{kg} / \mathrm{cm}^{2}$ & $0.986(0.933-1.043)$ & 0.628 & & \\
\hline Systolic BP, mmHg & $0.995(0.973-1.017)$ & 0.630 & & \\
\hline Diastolic BP, mmHg & $0.997(0.972-1.022)$ & 0.815 & & \\
\hline Heart rate, bpm & $1.005(0.983-1.029)$ & 0.651 & & \\
\hline NYHA class & $4.367(2.186-8.723)$ & $<0.001$ & $2.628(1.613-4.282)$ & $<0.001$ \\
\hline Pleural effusion & $5.604(2.334-13.456)$ & $<0.001$ & $0.810(0.356-1.845)$ & 0.616 \\
\hline Pericardial effusion & $0.970(0.432-2.180)$ & 0.942 & & \\
\hline \multicolumn{5}{|l|}{ Comorbidities } \\
\hline Diabetes mellitus & $1.235(0.462-3.301)$ & 0.674 & & \\
\hline Atrial fibrillation & $0.685(0.294-1.596)$ & 0.380 & & \\
\hline Coronary artery disease & $1.619(0.551-4.756)$ & 0.381 & & \\
\hline Intracardiac device & $1.544(0.530-4.494)$ & 0.426 & & \\
\hline \multicolumn{5}{|c|}{ Echocardiography parameters } \\
\hline LA length*, mm & $0.990(0.951-1.030)$ & 0.609 & & \\
\hline RA length*, mm & $1.004(0.963-1.047)$ & 0.854 & & \\
\hline LVEDD*, mm & $0.887(0.838-0.939)$ & $<0.001$ & $0.988(0.911-1.071)$ & 0.767 \\
\hline LVEF, \% & $1.010(0.976-1.046)$ & 0.556 & & \\
\hline LV-GLS, - \% & $1.113(1.020-1.215)$ & 0.017 & $1.133(1.018-1.261)$ & 0.022 \\
\hline IVS, mm & $1.115(1.024-1.214)$ & 0.013 & $1.071(0.943-1.215)$ & 0.291 \\
\hline RVEDD*, mm & $0.965(0.880-1.057)$ & 0.441 & & \\
\hline RV-TDI, m/s & $0.528(0.301-0.927)$ & 0.026 & $0.659(0.328-1.326)$ & 0.243 \\
\hline TAPSE, mm & $0.947(0.864-1.039)$ & 0.248 & & \\
\hline RV-GLS, - \% & $1.080(0.988-1.182)$ & 0.091 & & \\
\hline TR velocity, $\mathrm{m} / \mathrm{s}$ & $1.232(0.608-2.500)$ & 0.563 & & \\
\hline sPAP, mmHg & $1.011(0.983-1.040)$ & 0.428 & & \\
\hline IVC, $\mathrm{mm}$ & $0.958(0.877-1.046)$ & 0.335 & & \\
\hline \multicolumn{5}{|l|}{ Laboratory parameters } \\
\hline NT-pro BNP**pg/mL & $4.959(2.023-12.158)$ & 0.005 & $7.212(2.248-23.136)$ & 0.001 \\
\hline Troponin $\mathrm{T}^{* *} \mathrm{ng} / \mathrm{L}$ & $4.282(1.667-11.003)$ & 0.003 & $2.916(0.779-10.906)$ & 0.112 \\
\hline Hemoglobin, mg/dL & $0.996(0.833-1.192)$ & 0.968 & & \\
\hline Creatinine, mg/dL & $0.866(0.690-1.086)$ & 0.213 & & \\
\hline $\mathrm{eGFR}, \mathrm{mL} / \mathrm{min}$ & $0.992(0.978-1.005)$ & 0.241 & & \\
\hline ASAT, U/L & $1.008(0.997-1.020)$ & 0.154 & & \\
\hline ALAT, U/L & $1.010(0.997-1.022)$ & 0.139 & & \\
\hline Albumin, g/L & $0.968(0.930-1.008)$ & 0.113 & & \\
\hline GGT, U/L & $1.001(1.000-1.002)$ & 0.172 & & \\
\hline $\mathrm{LDH}, \mathrm{U} / \mathrm{L}$ & $1.003(1.000-1.006)$ & 0.080 & & \\
\hline $\mathrm{CRP}, \mathrm{mg} / \mathrm{dL}$ & $1.033(0.898-1.187)$ & 0.653 & & \\
\hline
\end{tabular}

$B M I$ body mass index, $B P$ blood pressure, $b p m$, beats per minute, NYHA New York Heart Association, $C A D$ coronary artery disease, $P M$ pace maker, $I C D$ intracardiac defibrillator, $A T I I$, angiotensin II, $A C E$ angiotensin converting enzyme, $L A$ left atrium, $R A$ right atrium, $L V E D D$ left ventricular end-diastolic diameter, $L V E F$ left ventricular ejection fraction, IVS intraventricular septum, RVEDD right ventricular end-diastolic diameter, $R V-T D I$ right ventricular tissue Doppler index, TAPSE tricuspid annular plane systolic excursion, $R V$-GLS right ventricular global longitudinal strain, $T R$ tricuspid regurgitation, $s P A P$ systolic pulmonary artery pressure, $I V C$ inferior vena cava, $N T$-pro $B N P \mathrm{~N}$-terminal pro brain natriuretic peptide, eGFR estimated glomerular filtration rate, ASAT aspartate aminotransferase, ALAT alanine aminotransferase, $G G T$ gamma glutamyltransferase, $L D H$ lactate dehydrogenase, $C R P C$-reactive protein

*Indexed to body surface area

**Log values were used for analysis 
the importance of assessment of pleural effusion as part of a standard echocardiography exam, as effusions may be too small to detect in chest X-ray.

\section{Limitations}

We are aware of the limitations, which are associated with the single-center design of the present study. However, this also implicates advantages regarding the consistency of diagnostic work-up, treatment and follow-up. Infiltration of pericardial or pleural tissue can of course not be certified without histological tissue characterization, which is not feasible in pre-mortem patient cohorts for obvious reasons. Even contrast CMR imaging would not be able to detect, let alone quantify amyloid deposits in the pericardium due to technical limitations. Effusion samples were not collected, because most patients did not undergo thoracocentesis, but were medically treated. Analysis of effusions could have given more insight into their underlying cause. Furthermore, we acknowledge, that diuretic treatment may have influenced the results of this study.

\section{Conclusion}

Pericardial and pleural effusions are present in more then $50 \%$ of patients with CA and assessment of effusion should be included in standard echocardiographic examination of all patients with suspected or definite diagnosis of CA. Pleural-, but not pericardial effusions were associated with adverse cardiac outcomes. The development of effusions in these patients may not simply be a sign of right heart failure, but may have a multifactorial genesis including decrease in serum albumin levels resulting in changes of oncotic pressures or local inflammation due do amyloid infiltration of the myocardium or pleural and pericardial serosae.

Acknowledgments Open access funding provided by Austrian Science Fund (FWF).

Funding This study was supported by the Medical Scientific Fund of the Mayor of the City of Vienna to Diana Bonderman (project number: 19011). This study received support from the Austrian Science Fund (FWF): KLI 246 (to D.B.)

Open Access This article is licensed under a Creative Commons Attribution 4.0 International License, which permits use, sharing, adaptation, distribution and reproduction in any medium or format, as long as you give appropriate credit to the original author(s) and the source, provide a link to the Creative Commons licence, and indicate if changes were made. The images or other third party material in this article are included in the article's Creative Commons licence, unless indicated otherwise in a credit line to the material. If material is not included in the article's Creative Commons licence and your intended use is not permitted by statutory regulation or exceeds the permitted use, you will need to obtain permission directly from the copyright holder. To view a copy of this licence, visit http://creativecommons.org/licenses/by/4.0/.

\section{References}

1. Natanzon A, Kronzon I (2009) Pericardial and pleural effusions in congestive heart failure-anatomical, pathophysiologic, and clinical considerations. Am J Med Sci 338:211-216. https://doi. org/10.1097/MAJ.0b013e3181a3936f

2. Grogan M, Scott CG, Kyle RA, Zeldenrust SR, Gertz MA, Lin G, Klarich KW, Miller WL, Maleszewski JJ, Dispenzieri A (2016) Natural history of wild-type transthyretin cardiac amyloidosis and risk stratification using a novel staging system. J Am Coll Cardiol 68:1014-1020. https://doi.org/10.1016/j.jacc.2016.06.033

3. Damy T, Jaccard A, Guellich A, Lavergne D, Galat A, Deux JF, Hittinger L, Dupuis J, Frenkel V, Rigaud C et al (2016) Identification of prognostic markers in transthyretin and AL cardiac amyloidosis. Amyloid Int J Exp Clin Investig 23:194-202. https ://doi.org/10.1080/13506129.2016.1221815

4. Adler Y, Charron P, Imazio M, Badano L, Baron-Esquivias G, Bogaert J, Brucato A, Gueret P, Klingel K, Lionis C et al (2015) 2015 ESC Guidelines for the diagnosis and management of pericardial diseases: the task force for the diagnosis and management of pericardial diseases of the European Society of Cardiology (ESC) endorsed by: The European Association for Cardio-Thoracic Surgery (EACTS). Eur Heart J 36:2921-2964. https://doi. org/10.1093/eurheartj/ehv318

5. Siepen FAD, Bauer R, Voss A, Hein S, Aurich M, Riffel J, Mereles D, Röcken C, Buss SJ, Katus HA et al (2018) Predictors of survival stratification in patients with wild-type cardiac amyloidosis. Clin Res Cardiol 107:158-169. https://doi.org/10.1007/s0039 2-017-1167-1

6. Soni NJ, Franco R, Velez MI, Schnobrich D, Dancel R, Restrepo MI, Mayo PH (2015) Ultrasound in the diagnosis and management of pleural effusions. J Hosp Med 10:811-816. https://doi. org/10.1002/jhm.2434

7. Ponikowski P, Voors AA, Anker SD, Bueno H, Cleland JGF, Coats AJS, Falk V, Gonzalez-Juanatey JR, Harjola VP, Jankowska EA et al (2016) 2016 ESC Guidelines for the diagnosis and treatment of acute and chronic heart failure: The Task Force for the diagnosis and treatment of acute and chronic heart failure of the European Society of Cardiology (ESC)Developed with the special contribution of the Heart Failure Association (HFA) of the ESC. Eur Heart J 37:2129-2200. https://doi.org/10.1093/eurhe artj/ehw128

8. Yuda S, Hayashi T, Yasui K, Muranaka A, Ohnishi H, Hashimoto A, Ishida T, Tsuchihashi K, Shinomura Y, Watanabe N et al (2015) Pericardial effusion and multiple organ involvement are independent predictors of mortality in patients with systemic light chain amyloidosis. Intern Med (Tokyo, Japan) 54:1833-1840. https://doi.org/10.2169/internalmedicine.54.3500

9. Escher F, Senoner M, Doerler J, Zaruba MM, Messner M, Mussner-Seeber C, Ebert M, Ensinger C, Mair A, Kroiss A et al (2020) When and how do patients with cardiac amyloidosis die? Clin Res Cardiol 109:78-88. https://doi.org/10.1007/s00392-019-01490-2

10. Kotecha T, Martinez-Naharro A, Treibel TA, Francis R, Nordin S, Abdel-Gadir A, Knight DS, Zumbo G, Rosmini S, Maestrini $\mathrm{V}$ et al (2018) Myocardial edema and prognosis in amyloidosis. J Am Coll Cardiol 71:2919-2931. https://doi.org/10.1016/j. jacc.2018.03.536

11. Chatzantonis G, Bietenbeck M, Florian A, Meier C, Stalling P, Korthals D, Reinecke H, Yilmaz A (2020) Diagnostic value of the novel CMR parameter "myocardial transit-time" (MyoTT) for the assessment of microvascular changes in cardiac amyloidosis 
and hypertrophic cardiomyopathy. Clin Res Cardiol. https://doi. org/10.1007/s00392-020-01661-6

12. Mishra S, Joshi S, Ward JE, Buys EP, Mishra D, Mishra D, Morgado I, Fisch S, Lavatelli F, Merlini G et al (2019) Zebrafish model of amyloid light chain cardiotoxicity: regeneration versus degeneration. American journal of physiology. Heart Circ Physiol 316:1158-1166. https://doi.org/10.1152/ajpheart.00788.2018

13. Ridouani F, Damy T, Tacher V, Derbel H, Legou F, Sifaoui I, Audureau E, Bodez D, Rahmouni A, Deux JF (2018) Myocardial native $\mathrm{T} 2$ measurement to differentiate light-chain and transthyretin cardiac amyloidosis and assess prognosis. J Cardiovasc Magn Reson 20:58. https://doi.org/10.1186/s12968-018-0478-3

14. Berk JL, Keane J, Seldin DC, Sanchorawala V, Koyama J, Dember LM, Falk RH (2003) Persistent pleural effusions in primary systemic amyloidosis: etiology and prognosis. Chest 124:969-977. https://doi.org/10.1378/chest.124.3.969

15. Gillmore JD, Maurer MS, Falk RH, Merlini G, Damy T, Dispenzieri A, Wechalekar AD, Berk JL, Quarta CC, Grogan M et al (2016) Nonbiopsy diagnosis of cardiac transthyretin amyloidosis. Circulation 133:2404-2412. https://doi.org/10.1161/circulatio naha.116.021612

16. Kammerlander AA, Duca F, Binder C, Aschauer S, Zotter-Tufaro C, Koschutnik M, Marzluf BA, Bonderman D, Mascherbauer J (2018) Extracellular volume quantification by cardiac magnetic resonance imaging without hematocrit sampling: ready for prime time? Wien Klin Wochenschr 130:190-196. https://doi. org/10.1007/s00508-017-1267-y

17. Duca F, Kammerlander AA, Panzenbock A, Binder C, Aschauer S, Loewe C, Agis H, Kain R, Hengstenberg C, Bonderman D et al (2018) Cardiac magnetic resonance T1 mapping in cardiac amyloidosis. JACC Cardiovasc Imaging 11:1924-1926. https:// doi.org/10.1016/j.jcmg.2018.06.010

18. Dispenzieri A, Lacy MQ, Katzmann JA, Rajkumar SV, Abraham RS, Hayman SR, Kumar SK, Clark R, Kyle RA, Litzow MR et al (2006) Absolute values of immunoglobulin free light chains are prognostic in patients with primary systemic amyloidosis undergoing peripheral blood stem cell transplantation. Blood 107:3378-3383. https://doi.org/10.1182/blood-2005-07-2922

19. Shah KB, Inoue Y, Mehra MR (2006) Amyloidosis and the heart: a comprehensive review. Arch Intern Med 166:1805-1813. https ://doi.org/10.1001/archinte.166.17.1805

20. Lang RM, Badano LP, Mor-Avi V, Afilalo J, Armstrong A, Ernande L, Flachskampf FA, Foster E, Goldstein SA, Kuznetsova $\mathrm{T}$ et al (2015) Recommendations for cardiac chamber quantification by echocardiography in adults: an update from the American Society of Echocardiography and the European Association of Cardiovascular Imaging. Eur Heart J Cardiovasc Imaging 16:233270. https://doi.org/10.1093/ehjci/jev014

21. Galderisi M, Cosyns B, Edvardsen T, Cardim N, Delgado V, Di Salvo G, Donal E, Sade LE, Ernande L, Garbi M et al (2017) Standardization of adult transthoracic echocardiography reporting in agreement with recent chamber quantification, diastolic function, and heart valve disease recommendations: an expert consensus document of the European Association of Cardiovascular Imaging. Eur Heart J Cardiovasc Imaging 18:1301-1310. https:// doi.org/10.1093/ehjci/jex244

22. Voigt JU, Pedrizzetti G, Lysyansky P, Marwick TH, Houle H, Baumann R, Pedri S, Ito Y, Abe Y, Metz S et al (2015) Definitions for a common standard for 2D speckle tracking echocardiography: consensus document of the EACVI/ASE/Industry Task Force to standardize deformation imaging. J Am Soc Echocardiogr 28:183-193. https://doi.org/10.1016/j.echo.2014.11.003

23. Binder C, Duca F, Stelzer PD, Nitsche C, Rettl R, Aschauer S, Kammerlander AA, Binder T, Agis H, Kain R et al (2019) Mechanisms of heart failure in transthyretin vs. light chain amyloidosis. Eur Heart J Cardiovasc Imaging 20:512-524. https://doi. org/10.1093/ehjci/jey225

24. Kreusser MM, Volz MJ, Knop B, Ehlermann P, Schmack B, Ruhparwar A, Hegenbart U, Schönland SO, Katus HA, Raake PW (2020) A novel risk score to predict survival in advanced heart failure due to cardiac amyloidosis. Clin Res Cardiol 109:700-713. https://doi.org/10.1007/s00392-019-01559-y 